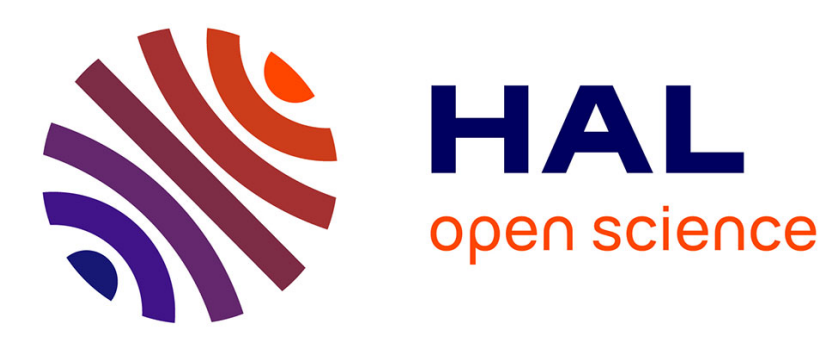

\title{
Quadratic spatial soliton generation by down conversion of a strong harmonic pump beam
}

Michael Canva, R.A. Fuerst, D. Baboiu, George I. Stegeman, G. Assanto

\section{To cite this version:}

Michael Canva, R.A. Fuerst, D. Baboiu, George I. Stegeman, G. Assanto. Quadratic spatial soliton generation by down conversion of a strong harmonic pump beam. Optics Letters, 1997, 22 (22), pp.1683-1685. hal-00668805

\section{HAL Id: hal-00668805 \\ https://hal-iogs.archives-ouvertes.fr/hal-00668805}

Submitted on 10 Feb 2012

HAL is a multi-disciplinary open access archive for the deposit and dissemination of scientific research documents, whether they are published or not. The documents may come from teaching and research institutions in France or abroad, or from public or private research centers.
L'archive ouverte pluridisciplinaire HAL, est destinée au dépôt et à la diffusion de documents scientifiques de niveau recherche, publiés ou non, émanant des établissements d'enseignement et de recherche français ou étrangers, des laboratoires publics ou privés. 


\title{
Quadratic spatial soliton generation by seeded downconversion of a strong harmonic pump beam
}

\author{
M. T. G. Canva, R. A. Fuerst, S. Baboiu, and G. I. Stegeman \\ Center for Research and Education in Optics and Lasers, University of Central Florida, 4000 Central Florida Boulevard, \\ Orlando, Florida 32816-2700 \\ G. Assanto \\ Department of Electronic Engineering, Terza University of Rome, 84-00146 Rome, Italy
}

Received July 29, 1997

\begin{abstract}
Two-dimensional quadratic spatial solitons were generated experimentally near phase-matching conditions for type II frequency doubling in KTP by the seeding of a strong second-harmonic field with a weak input at the fundamental wavelength. The self-trapped beams were shown to be insensitive to the energy, phase, and polarization of the fundamental-frequency seed input beam. (c) 1997 Optical Society of America
\end{abstract}

Quadratic solitons, which can be generated during second-harmonic generation (SHG), have been investigated in geometries of various dimensionalities and for various types of phase matching, most specifically type I and type II. ${ }^{1-8}$ These solitons consist of waves at multiple frequencies that propagate locked together in space owing to their strong coupling via secondorder nonlinearities. ${ }^{2}$ It is understood that these solitons can be generated routinely during SHG under appropriate but easy to implement conditions, and, by extension, they should be pervasive in other second-order nonlinear interactions. One especially interesting case occurs in downconversion interactions, which are at the core of generating new frequencies in parametric oscillators and amplifiers. ${ }^{9}$ A simple example is the degeneracy point of a parametric generator that produces two photons of equal frequency from one pump photon when the wave-vector-matching condition among the three waves, i.e., the reverse of SHG, is satisfied. Usually this condition occurs as a parametric instability; i.e., above a threshold intensity this process is triggered by noise, and a resonator is used to allow the downconverted beams to grow and efficient conversion to occur. However, this process can also be triggered by a seed beam at half the pump frequency and leads to the generation of quadratic solitons, as recently predicted by Leo and Assanto. ${ }^{10}$ They found that the creation of downconverted quadratic solitons is a very robust process that occurs under a variety of seeding conditions and is potentially useful for all-optical switching.

This Letter describes the experimental formation of quadratic spatial solitons (QSS's) in a type II quadratic interaction between a strong second-harmonic pump field $(\mathrm{PF})$ and a seed of one of the corresponding frequency-degenerate fundamental signal fields (SF's). In the material system under investigation, potassium titanyl phosphate (KTP), the extraordinarily polarized harmonic field interacts with two fundamental fields with both ordinary and extraordinary components (eoe in the usual SHG notation). The weak SF initiates the downconversion of the strong PF, and energy is interchanged among the three fields. When a strong enough $\mathrm{PF}$ is injected into the crystal, this interaction can lead to the formation of a stable, nondiffracting three-coupled-wave system. This system is actually a QSS similar to those obtained through initial upconversion by Torruellas et al. when only fundamental waves were launched. ${ }^{5}$

The experiments were performed in an $L=1 \mathrm{~cm}$ KTP crystal cut for type II phase matching in the $X-Y$ plane. The experimental setup was similar to the one in Ref. 5 but with an additional arm for the extra wavelength. The source was a $Q$-switched mode-locked Nd:YAG laser that provided 35-ps pulses of several tens of microjoules at $10 \mathrm{~Hz}$ at a wavelength of $1064 \mathrm{~nm}$. A type I KD*P crystal was used to double the laser frequency to provide a strong pump field. A dichroic mirror was used to separate this second harmonic $(\mathrm{PF})$ from the copropagating fundamental wave (SF) so that each beam could be controlled independently with respect to its size (both were $\sim 20 \mu \mathrm{m}$ in diameter), intensity, and polarization ( $e$ for the $\mathrm{PF}$ and a variable $o-e$ ratio for the SF). Strong absorption filters $\left(T \approx 10^{-21}\right.$ at $\left.1064 \mathrm{~nm}\right)$ removed any residual fundamental photons from the PF. The temporal overlap of the green and the IR pulses was verified with a 2-ps-resolution streak camera. The KTP crystal was placed upon a rotation stage so that the effects of phase mismatch could be studied. The output beam profile at various planes throughout the propagation was imaged with $37 \times$ magnification into a video camera sensitive to the IR. Additional measurements of the output beams were performed with a $1-\mathrm{cm}^{2}$ pyroelectric energy meter.

First the PF pulse energy threshold for QSS formation within the crystal (five diffraction lengths long) was evaluated for various phase mismatches with a fixed amount of SF seed. Soliton formation was confirmed on the camera when the output fundamental beam diameter collapsed from $\sim 100 \mu \mathrm{m}$ at low intensities to $\sim 15 \mu \mathrm{m}$ above threshold. For SF pulses of $\sim 15 \mathrm{~nJ}$, the PF pulse energy threshold for formation of a QSS was measured and is plotted versus phase mismatch $\Delta \beta$ in Fig. 1. Here $\Delta \beta=\left(k_{1}+k_{2}-k_{3}\right) L$, where the $k_{i}$ are the wave vectors associated with the SF's $e$ - and $o$-polarized components $(i=1$ and $i=2$, respectively) and the $e$-polarized $\mathrm{PF}(i=3)$. 


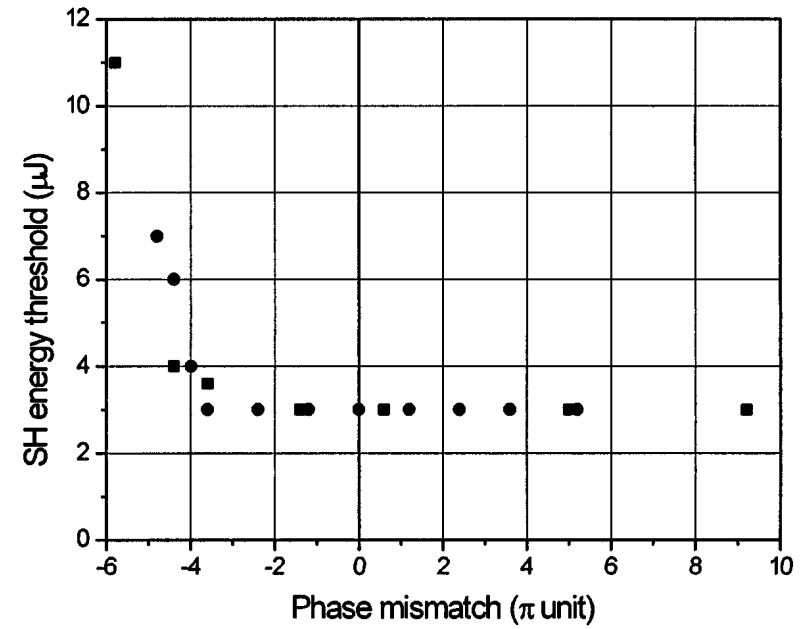

Fig. 1. Second-harmonic PF energy threshold for the formation of the QSS in our experimental conditions for different detunings $\Delta \beta$; the SF energy is $15 \mathrm{~nJ}$.

The trends are intuitive in light of the self-focusing and self-defocusing that occurred away from phase matching. ${ }^{1} \quad$ Lower intensities are required for balancing of diffraction for a self-focusing mismatch $(\Delta \beta>0)$, whereas larger intensities are needed for negative selfdefocusing mismatch $(\Delta \beta<0)$. It was also observed that the threshold and the QSS profile were sharper in the case of negative $\Delta \beta$.

Since downconversion can be seeded by quantum noise within the crystal, a spatial soliton could be generated if a strong enough PF were launched into the crystal and a suitable number of diffraction lengths were available for propagation, for example, in a resonator. However, here the optical path was limited to only five diffraction lengths, and because KTP possesses a moderate damage threshold the PF energy launched into the crystal was limited to tens of microjoules. Therefore a threshold value for the SF seed was required for soliton formation. The effects of variations in SF energy on the resulting output energy and the output energy in the harmonic (ex-pump) and the fundamental (ex-signal) frequencies are illustrated in Fig. 2. For $\Delta \beta=-2 \pi$ and a fixed PF input energy of $4 \mu \mathrm{J}$, SF energies of more than $1 \mathrm{pJ}$ lead to the formation of a QSS. Increasing this SF energy further produces a plateau region in which the IR and the green energies leaving the crystal are relatively constant. This result shows that, for a wide range of SF inputs, the formation of the QSS is independent of seed power and is thus characterized by phase mismatch and pump power. Qualitatively, the soliton composition (harmonic versus fundamental frequencies) is maintained over several orders of magnitude in incident seed energy, with a fixed energy throughput. Only when the IR seed energy exceeds a few tens of nanojoules do the output characteristics change. It was observed that, above these levels, the additional SF energy led to higher throughputs even though the harmonic output energy remained approximately constant. Moreover, as the phase mismatch varied from negative to increasing positive values, the $\mathrm{SF}$ input threshold for a QSS increased, as did the fraction of the output fundamental-frequency component. In all cases plateaus at which the soliton output is independent of the input SF energy were observed over several orders of magnitude. Thus a wide family of QSS's differing by their relative ratios of harmonic to fundamental power was observed for different detuning conditions. Changing their power also affected their internal imbalance.

Next the sensitivity of soliton formation to the polarizations and the phases of the seed beams was investigated for fixed values of PF energy $(4 \mu \mathrm{J})$, above PF threshold, and with fixed SF energy (15 nJ), within the plateau of Fig. 2. Soliton generation was found to be independent of the polarization of the weak SF seed; i.e., QSS's were observed for all possible polarization states of the input seed when these states were imaged into the camera. More quantitatively, the total energy and the ratio of the fundamental to the second-harmonic components trapped in the QSS and its surroundings were constant to within $10 \%$ for all polarization states. The dependence of the QSS formation on the relative delay between the $\mathrm{PF}$ and the SF fields (adjusted by a delay line in the PF arm of the experimental setup) was also determined under the same conditions. The fundamental and the harmonic outputs did not vary significantly with relative delay. For example, at $0 \pi$ detuning the output components were almost equal at approximately $1.2 \mu \mathrm{J}$ (a total output of $2.4 \mu \mathrm{J}$ was produced by an input launch of $4 \mu \mathrm{J}$ of PF energy). However, a slight drop occurred at a delay of $\pm 70 \mathrm{ps}$ (i.e., twice the pulse width). At this point the overlap between the $\mathrm{PF}$ and the SF pulses is insufficient to form a QSS (i.e., the overlap is below threshold). Similarly, it was observed that the spatial overlap of the PF and the SF beams was not critical. Misaligning the PF and SF spots focused on the front face of the crystal by several beam widths did not seem to hinder spatial soliton formation, as one would expect considering that only a very small $\mathrm{SF}$ is actually required for the formation of the QSS. ${ }^{10}$

A final investigation of the composition of the QSS as a function of phase mismatch was performed. A fixed SF seed of $15 \mathrm{~nJ}$ and a PF pulse energy of first 4 and then $8 \mu \mathrm{J}$ allowed us to perform the experiments above

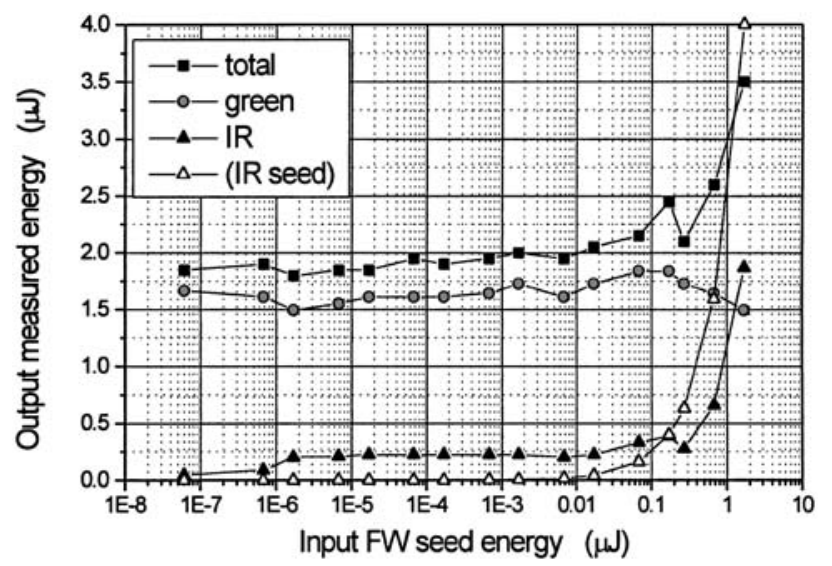

Fig. 2. SF energy effect on the output fundamental wave (FW) and the harmonic wave in our experimental conditions; the PF energy is $4 \mu \mathrm{J}$, and the detuning $\Delta \beta$ is $-2 \pi$. 

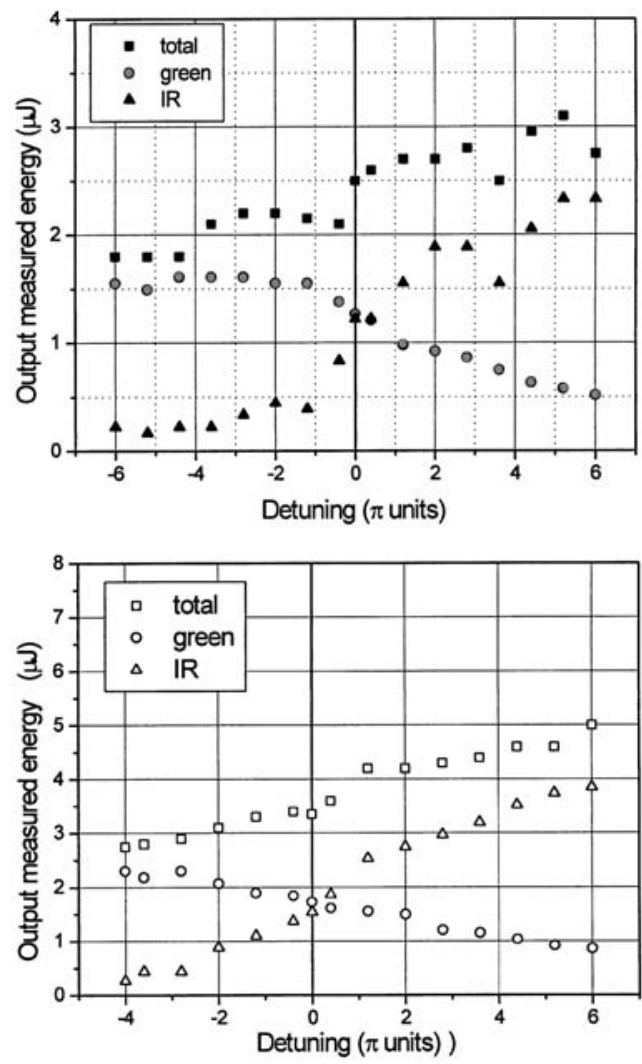

Fig. 3. Output structure, i.e., fundamental and harmonic energies, measured for different detuning $\Delta \beta$ conditions; at the input the harmonic PF was (top) $4 \mu \mathrm{J}$ and (bottom) $8 \mu \mathrm{J}$, and the fundamental SF was $15 \mathrm{~nJ}$.

threshold for nearly all mismatches between $-6 \pi$ and $+6 \pi$. The output energies at both wavelengths were measured as a function of detuning and are shown in Fig. 3. At phase matching the energy components of the QSS and its surroundings are nearly equal, 50\% energy at each frequency. It is again important to note that the quadratic solitons vary dramatically in their composition with phase mismatch. For $4 \mu \mathrm{J}$ of $\mathrm{PF}$ energy and increasing positive wave-vector mismatch, the fundamental-frequency component becomes progressively more dominant, from $20 \%$ at $\Delta \beta=6 \pi$ to $80 \%$ for $\Delta \beta=+6 \pi$. When the PF energy was increased by $100 \%$ to $8 \mu \mathrm{J}$, the same trend was followed but only an extra $25 \%$ of energy was trapped (75\% of the extra $4 \mu \mathrm{J}$ of energy did not make it to the large detector), whereas the initial trapping was $\sim 66 \%$ for the previously launched $4 \mu \mathrm{J}$ of energy. Moreover, for an 8- $\mu \mathrm{J}$ input energy value the variations with detuning are more linear than in the $4-\mu \mathrm{J}$ case, in which one can see a kind of saturation on the negative side as the energy threshold is crossed at $-4 \pi$. Also, for a given detuning, a higher PF energy tends to increase the harmonic-fundamental imbalance.

In addition to the switching applications originally envisaged by Leo and Assanto, this seeded parametric instability may have an application as an all-optical power transistor with a clamped output. Here the pump beam works as a power supply. As shown in Fig. 2, signal input ranging over 4 orders of magnitude produced a constant signal output, corresponding to gains of $G=10^{5}$ to $G=10$, even adapting to $G=1$ on the next order of magnitude of variation of the increasing SF energy. This feature is a direct consequence of the exponential growth of the instability combined with the generation of a nonlinear eigenmode (the soliton). In addition, the signal output is independent of the polarization of the input signal, and the noise on the output is determined primarily by the pump beam (power supply) noise. Finally, we note that the temporal and spatial overlap of the input signal with the pump beam are not critical for efficient operation.

In summary, it has been verified experimentally that parametric downconversion leads to quadratic soliton generation at the degeneracy point (and presumably elsewhere). This finding was demonstrated for a strong pump at $532 \mathrm{~nm}$ in type II KTP, seeded at the fundamental wavelength (for the corresponding SHG process). In a 1-cm-long crystal, soliton formation was observed above a threshold seed energy and over several orders of magnitude. The process was also found to be essentially independent of the polarization of the input seed. Consequently, the temporal and spatial overlap of the two input beams was demonstrated to be unnecessary within reason (several times the pulse dimension). In addition to all-optical switching, this quadratic soliton formation process is of more fundamental interest in parametric processes such as optical parametric oscillators and optical parametric amplifiers. Finally, it is clear that a broad spectrum of quadratic spatial solitons can be generated with different fractional compositions of fundamental and harmonic fields, depending on the input and detuning conditions.

This research was supported in the United States by the National Science Foundation, the U.S. Air Force Office of Scientific Research, the Defense Advanced Research Projects Agency, and the U.S. Army Research Office. M. Canva acknowledges support from the French Direction Generale de l'Armament under contract ERE 96-1101. We thank Giuseppe Leo for fruitful discussions.

\section{References}

1. Reviewed in G. Stegeman, D. Hagan, and L. Torner, J. Opt. Quantum. Electron. 28, 1691 (1996).

2. Yu. Karamzin and A. Sukhorukov, Zh. Eksp. Teor. Phys. 68, 834 (1975) [Sov. Phys. JETP 41, 414 (1976)].

3. D. Pelinovsky, A. Buryak, and Y. Kivshar, Phys. Rev. Lett. 75, 591 (1995).

4. L. Torner, C. Menyuk, W. Torruellas, and G. I. Stegeman, Opt. Lett. 20, 13 (1995).

5. W. Torruellas, Z. Wang, D. Hagan, E. Van Stryland, G. Stegeman, L. Torner, and C. Menyuk, Phys. Rev. Lett. 74, 5036 (1995).

6. R. Schiek, Y. Baek, and G. Stegeman, Phys. Rev. E 53, 1138 (1996).

7. R. Fuerst, B. Lawrence, W. Torruellas, and G. Stegeman, Opt. Lett. 22, 19 (1997).

8. R. Fuerst, M. Canva, D. Baboiu, and G. Stegeman, "Properties of type II quadratic solitons excited by fundamental waves," Opt. Lett. (to be published).

9. M. Werner and P. Drummond, Opt. Lett. 19,613 (1994).

10. G. Leo and G. Assanto, Opt. Lett. 22, 1391 (1997). 